Original

\title{
Estado actual de Melongena melongena (Mollusca: Gastropoda) en la bahía de Cispatá, Caribe Colombiano
}

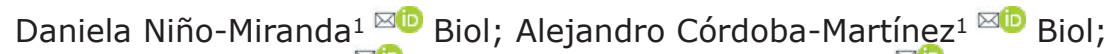 \\ Luz Arias-Reyes ${ }^{\otimes(\mathbb{E})}$ Esp; Jorge Quirós-Rodríguez ${ }^{*} \bowtie \mathbb{B}$ M.Sc.
}

\begin{abstract}
1 Universidad de Córdoba, Facultad de Ciencias Básicas, Departamento de Biología, Montería, Colombia.
¿Universidad de Córdoba, Facultad de Medicina Veterinaria y Zootecnia, Departamento de Ciencias Acuícolas, Montería, Colombia.

3Universidad de Córdoba, Facultad de Ciencias Básicas, Departamento de Biología, Grupo de Investigación Química de los Productos Naturales: PRONAT, Montería, Colombia.
\end{abstract}

*Correspondencia: alexander_quiroz@hotmail.com

Recibido: Noviembre 2019; Aceptado: Mayo 2020; Publicado: Julio 2020.

\section{RESUMEN}

Objetivo. Evaluar el estado actual de Melongena melongena en la bahía de Cispatá, Caribe Colombiano a través de los aspectos poblacionales de la especie. Materiales y métodos. Se establecieron tres sectores de muestreo, conforme con la zonificación de los manglares en la bahía. Para la recolección del material biológico se ubicó un transecto lineal de $4 \times 20 \mathrm{~m}$ perpendicular al área del manglar con dos replicas a una distancia de $50 \mathrm{~m}$. Para la determinación del sexo, se tomó una submuestra de 10 individuos con tallas superiores a la estimada para la madurez sexual de la especie. A las conchas se les midió la longitud total, con el fin de diferenciar las tallas de hembras y machos. En cada sector, la temperatura del agua y salinidad fueron registradas in situ, empleando un medidor multiparámetro Extech EC170, mientras la trasparencia del agua se calculó mediante el disco Secchi. Resultados. Se registraron un total de 1.149 individuos de M. melongena, obteniendo la mayor abundancia el sector Caño Salado (522), seguido de Las Cagás (458) y finalmente Amaya (169). El $63 \%$ de los individuos se encontraron en un intervalo de talla entre 41-61 mm. La proporción sexual fue 1:1.2 ( $\mathrm{H}: \mathrm{M})$. Las tallas registradas en hembras fueron de 55 a $92 \mathrm{~mm}$ y en machos de 54 a 77 $\mathrm{mm}$. Conclusiones. M. melongena en la bahía de Cispatá está presentando signos evidentes de recuperación en la última década, debido a que la mayoría de los individuos registrados han alcanzado la talla media de madurez sexual.

Palabras Clave: Abundancia; caracol; estuario; manglar; pesquería; proporción sexual; talla (Fuente: Tesauro Ambiental para Colombia).

\section{ABSTRACT}

Objective. Evaluate the current status of Melongena melongena in Cispata Bay, Colombian Caribbean through the population aspects of the species. Materials and methods. Three sampling sectors were established, according to the zoning of the mangrove in the bay. For the collection of the biological

Como citar (Vancouver).

Niño-Miranda D. Córdoba-Martínez A, Arias-Reyes L, Quirós-Rodríguez J. Estado actual de Melongena melongena (Mollusca: Gastropoda) en la bahía de Cispatá, Caribe Colombiano. Rev MVZ Cordoba. 2020; 25(2):e1873. https://doi.org/10.21897/rmvz.1873

CEl (los) autor (es), Revista MVZ Córdoba 2020. Este artículo se distribuye bajo los términos de la licencia internacional Creative Commons Attribution CC) $\$ 4.0$ (https://creativecommons.org/licenses/by-nc-sa/4.0/), que permite a otros distribuir, remezclar, retocar, y crear a partir de su obra de modo no BY NC SA comercial, siempre y cuando den crédito y licencien sus nuevas creaciones bajo las mismas condiciones. 
material a linear transect of $4 \times 20 \mathrm{~m}$ was located perpendicular to the area of the mangrove with two replicas at a distance of $50 \mathrm{~m}$. For the determination of sex, a subsample of 10 individuals with sizes greater than that estimated for the sexual maturity of the species was taken. The shells were measured in total length, in order to differentiate the sizes of females and males. In each sector, the water temperature and salinity were measured in situ using an Extech EC170 multiparameter while water transparency is calculated using the Secchi disk. Results. A total of 1,149 individuals of M. melongena were recorded, obtaining the highest abundance in the Caño Salado sector (522), followed by Las Cagás (458) and finally Amaya (169). 63\% of the individuals were in a size range between 41-61 mm. The sex ratio was 1:1.2 (H:M). The sizes recorded in females were from 55 to $92 \mathrm{~mm}$ and in males from 54 to $77 \mathrm{~mm}$. Conclusions. M. melongena in Cispatá Bay is showing evident signs of recovery in the last decade, because most of the registered individuals have reached the average size of sexual maturity.

Keywords: Abundance; estuary; fishery; mangrove; sexual proportion; size; snail (Source: Environmental thesaurus for Colombia).

\section{INTRODUCCIÓN}

Melongena melongena (L.) se distribuye ampliamente en el Caribe, las Antillas y el golfo de México. Este caracol vive en las zonas poco profundas de hábitat marinos, estuarinos y costeros, asociado a los manglares, siendo un componente importante de la fauna en fondos blandos o fangosos. La especie tiende a concentrarse en áreas con altas densidades de su presa, que corresponden a bivalvos, otros caracoles, ascidias y carroña $(1,2,3,4)$. Teniendo en cuenta este comportamiento, los pescadores a veces usan las raíces de manglar, cubiertas con bivalvos como carnada (5). Este caracol alcanza tamaños de hasta $200 \mathrm{~mm}$ de altura de concha (6), siendo la Talla Media de Madurez (TMM) más temprana en los machos (52 $\mathrm{mm}$ ) que en las hembras $(65 \mathrm{~mm})$ de acuerdo con los resultados obtenidos por Hernández y Stotz (5).

En Colombia M. melongena se ha convertido en un recurso alimenticio sustancial capturándose artesanalmente a través de la colecta manual, principalmente para autoconsumo o comercialización local y regional (7), por lo que la especie está siendo capturada por debajo de su TMM y su pesquería se está ejerciendo sobre tallas pequeñas, debido a que el método de captura utilizado por los pescadores no es selectivo (8). Lo cual, ha llevado a que esta población presente signos de sobreexplotación y por consiguiente una disminución de su producción en ciertas áreas del Caribe Colombiano $(5,9)$. A pesar de su importancia económica, y de ser una especie de fondos someros en zonas del manglar, las investigaciones acerca de esta especie son escasas. La descripción morfológica y distribución geográfica de $M$. melongena en el Caribe Colombiano se encuentra en Díaz y Puyana (6), mientras que estudios sobre algunos aspectos biológicos y pesqueros de la especie en el complejo estuarino de Cispatá se muestran en Hernández y Stotz (5), quienes además registraron la TMM conocida para este caracol hasta el momento.

El objetivo de la presente investigación fue actualizar la información M. melongena en la bahía de Cispatá centrada en su abundancia, talla y proporción sexual, así como la relación de esta especie con algunas variables ambientales como la temperatura, salinidad y transparencia, con el fin de contribuir con información científica que permita establecer por parte de las entidades correspondientes las medidas de manejo y conservación de este recurso.

\section{MATERIALES Y MÉTODOS}

Área de estudio. La bahía de Cispatá se sitúa en el sur del golfo de Morrosquillo, entre las coordenadas $09^{\circ} 22^{\prime} 07^{\prime \prime}, 09^{\circ} 26^{\prime} 11^{\prime \prime}$ latitud norte y 7546'30", 7556'30" longitud oeste (Figura 1), hace parte del Distrito de Manejo Integrado (DMI) de Cispatá, que comprende una extensión total de manglar de 8.571 ha (10). El régimen climático en la zona es unimodal-biestacional, con una estación seca bien definida de diciembre a abril, y una húmeda de mayo a noviembre que coincide con el aumento del caudal del río Sinú. La precipitación promedio anual es de $1337.4 \mathrm{~mm}$, la evaporación potencial promedio es de 1.826 $\mathrm{mm} / \mathrm{año} \mathrm{y}$ la temperatura promedio anual es de $28^{\circ} \mathrm{C}$, oscilando entre 32.9 y $26.7^{\circ} \mathrm{C}(8,10)$. 


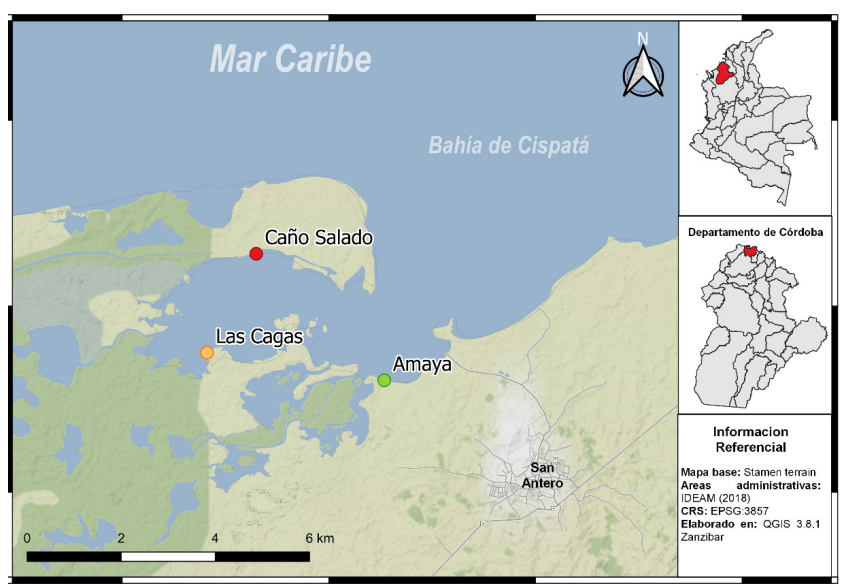

Figura 1. Área de estudio y ubicación de los sectores de muestreo en la bahía de Cispatá, departamento de Córdoba, Caribe Colombiano. Círculo rojo: Caño Salado, círculo amarillo: Las Cagas, círculo verde: Amaya.

Descripción de los sectores. Se establecieron tres sectores de muestreo, conforme con la zonificación de los manglares en la bahía, las cuales consisten en divisiones espaciales y geográficas, según principios de agrupamiento de índoles ecológico, social, económico y logístico (10). Se monitoreó de marzo a octubre de 2016 la zona de preservación, uso sostenible y recuperación.

Caño Salado $\left(09^{\circ} 41^{\prime} 84^{\prime \prime} \mathrm{N}, 75^{\circ} 81^{\prime} 48^{\prime \prime} \mathrm{O}\right)$ se caracteriza por ser un sector que esta desconectado del río Sinú y solo recibe agua en inundaciones muy fuertes; corresponde a una zona de preservación de los manglares, que por su importancia ecológica, alta productividad, ubicación estratégica y buen estado de conservación debe ser protegida para la investigación, la educación y la conservación de especies y comunidades (10); Las Cagas $\left(09^{\circ} 39^{\prime} 70^{\prime \prime} \mathrm{N}, 7^{\circ} 82^{\prime} 32^{\prime \prime} \mathrm{O}\right)$ se encuentra constituido por un bosque maduro y mixto, principalmente de Rhizophora mangle, Conocarpus erectus y Laguncularia racemosa. Se encuentra dentro de la zona de uso sostenible, que son áreas de manglar susceptibles de uso directo y sostenible como modalidad de conservación, en esta zona los niveles de aprovechamiento nunca podrán ser mayores a los ciclos de reproducción y crecimiento de los recursos usados o extraídos

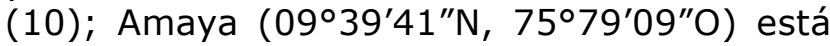
caracterizada por sus fondos areno fangosos, corresponde a una zona de recuperación de las áreas de manglar, que son áreas que debido a su mal estado de conservación o estado progresivo de degradación requieren de acciones encaminadas a recuperar bienes y servicios (10).
Fase de campo. En cada sector, la temperatura del agua y salinidad fueron registradas in situ, empleando un medidor multiparámetro Extech EC170. La estimación de la trasparencia del agua se realizó mediante el empleo del disco Secchi. Para la recolección del material biológico se ubicó un transecto lineal de $4 \times 20$ $\mathrm{m}$ perpendicular al área del manglar con dos replicas a una distancia de $50 \mathrm{~m}$, estableciéndose tres estratos con relación a la distancia al manglar $(5,11)$. A través de buceo autónomo en cada transecto se ubicaron al azar diez cuadrantes de $1 \mathrm{~m}^{2}$, donde se contabilizaron y midieron en términos de su longitud total todos los individuos de M. melongena. Para la determinación del sexo, en cada muestreo se recolectó una submuestra de 10 individuos con tallas superiores a la estimada para la madurez sexual de la especie (5). Luego, el material fue debidamente preservado, almacenado y transportado al Laboratorio de Postcosecha de la Universidad de Córdoba (LPUC).

Fase de laboratorio. Los ejemplares recolectados fueron sometidos a calentamiento durante cinco minutos con el fin de separar el tejido blando de la concha. Posterior a esto, se observó al estereoscopio la presencia de pene en los machos y oviducto en hembras. Finalmente, a las conchas se les midió la longitud total, con el objeto de diferenciar las tallas de hembras y machos. Los especímenes de referencia fueron depositados en el Laboratorio de Zoología de la Universidad de Córdoba (LZUC-MOL 00105, 00106, 00107).

Análisis de los datos. La densidad se calculó dividiendo el número total de caracoles recolectados por el total de metros muestreados (caracoles/ $\mathrm{m}^{2}$ ). Esta operación se realizó con el total de caracoles recolectados en todos los transectos. Se evaluaron los supuestos de normalidad con el test de Shapiro-Wilks, posteriormente se realizó una prueba de Kruskall-Wallis $(\mathrm{H})$, con el fin de determinar si existen diferencias significativas entre el número de especímenes en los sectores y meses de muestreo.

La estructura de tallas para la especie se determinó mediante el uso de histogramas de frecuencia de talla $(\mathrm{mm})$, se eligieron intervalos de $20 \mathrm{~mm}$ con base en la talla mínima y máxima observada para la especie, tomando como referencia el trabajo realizado por Hernández y Stotz (5). Para conocer la proporción sexual, se dividió el total de hembras recolectadas por el número total de machos (F:M). Este cálculo 
se realizó sobre la base del total de hembras y machos que se recogieron en todos los muestreos y el total por cada área muestreada en los tres sectores. Se comparó mediante la prueba de Chicuadrado $\left(\mathrm{X}^{2}\right)$, la proporción del número total de machos y hembras en todo el estudio.

Finalmente, para determinar que variables fisicoquímicas fueron más determinantes de los cambios en la abundancia del caracol en la bahía de Cispatá, se realizó un análisis de redundancia (RDA) usando el programa $R$ versión 3.6.1. Para esto se utilizaron los datos de las abundancias de los individuos del caracol en cada sector y mes de muestreo. En el RDA las variables fisicoquímicas registradas en campo, se les determinó su significancia con el test de Monte Carlo $(p<0.05)$.

\section{RESULTADOS}

Variables ambientales. La salinidad media superficial fue menor en octubre en Amaya (19.1 ups) y Caño Salado (15.2 ups) debido al efecto de las lluvias y alta en marzo y abril para cada sector (35 ups) por efecto de la evaporación. La salinidad promedio fue similar en Amaya (26.6 ups) y Caño Salado (26.3 ups) durante ese año, mientras que en Las Cagas fue más alta (28.1 ups). La temperatura media del agua en cada sector fue mayor en los meses de abril, mayo, septiembre y octubre a causa de los periodos lluviosos del año, y baja en junio y julio $\left(<27^{\circ} \mathrm{C}\right)$ debido al descenso de las precipitaciones como consecuencia del veranillo de San Juan. La transparencia del agua tuvo un mínimo de 66.7 $\mathrm{cm}$ en octubre y un máximo de $91.7 \mathrm{~cm}$ en marzo, tendencia que se reflejó en cada una de las localidades muestreadas (Figura 2).
A
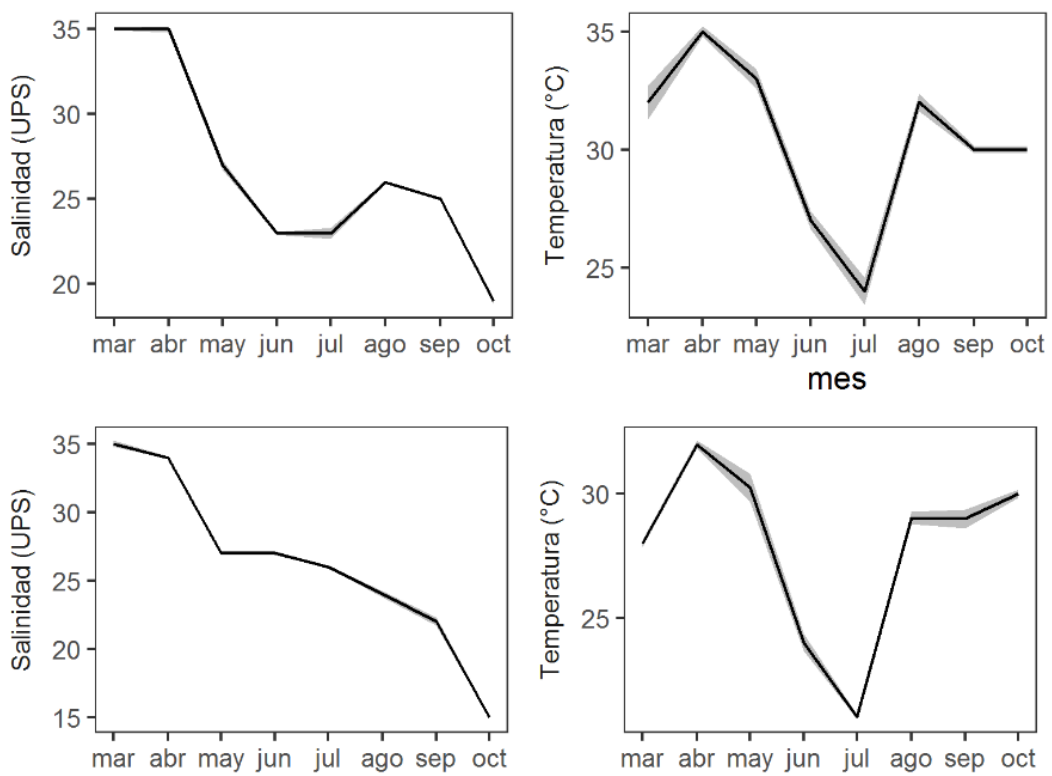

B

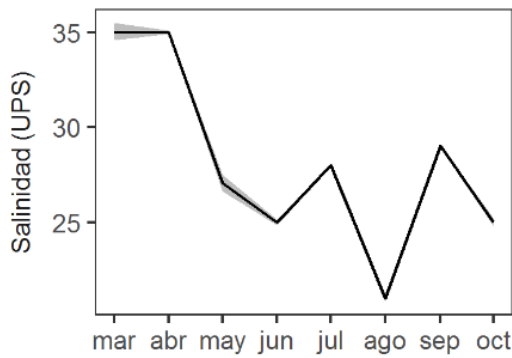

C
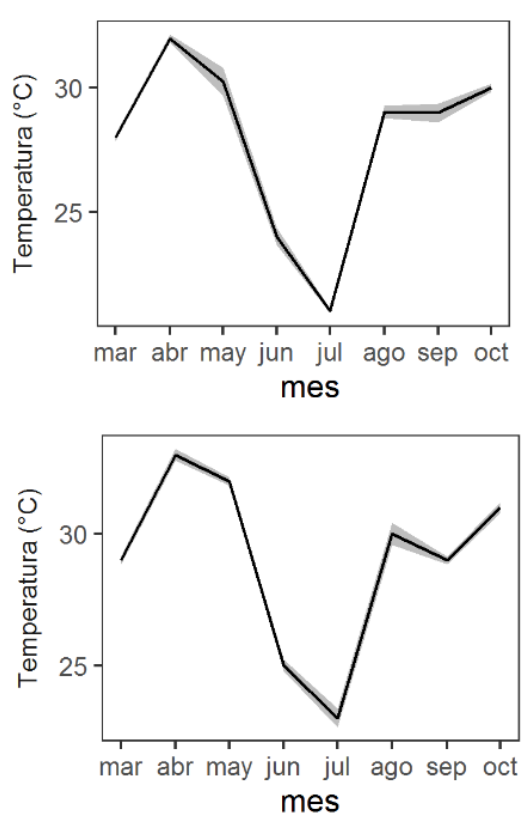
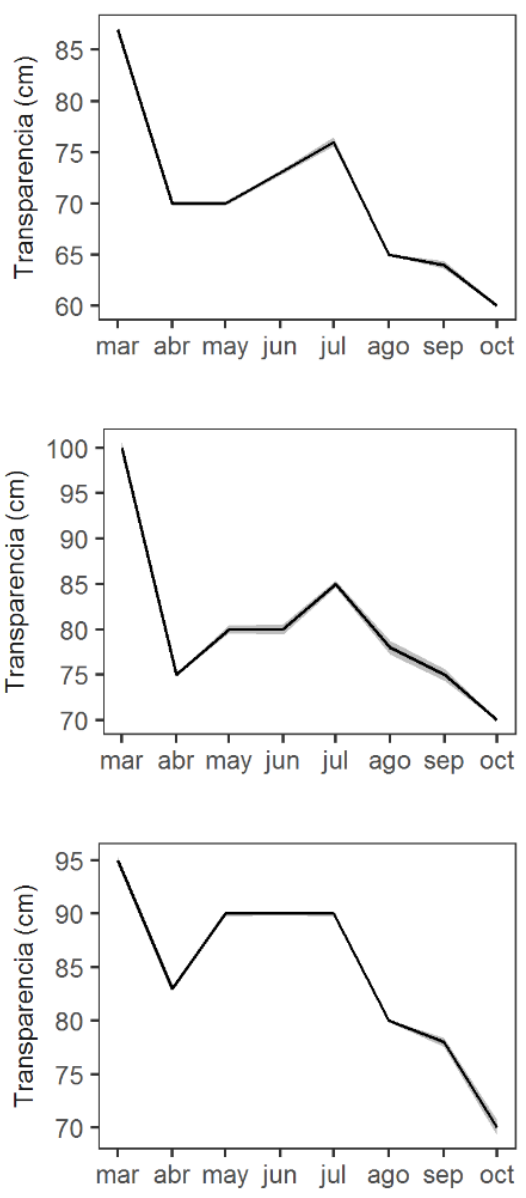

Figura 2. Fluctuaciones de los valores promedios ambientales (salinidad, temperatura y transparencia) en la bahía de Cispatá de marzo a octubre de 2016. A. Amaya, B. Caño Salado, C. Las Cagas 
Abundancia de $M$. melongena. Se registró un total de 1.149 individuos en el área de estudio (14.36 ind $/ \mathrm{m}^{2}$ ) donde las mayores densidades se registraron en el sector de Caño Salado $\left(6.5 \mathrm{ind} / \mathrm{m}^{2}\right)$ y las Cagás $\left(5.7 \mathrm{ind} / \mathrm{m}^{2}\right)$, y las menores densidades en el sector Amaya (2.1 ind $/ \mathrm{m}^{2}$ ) (Figura 3A). En marzo, abril y mayo, se reportaron los mayores valores de abundancia promedio, mientras que los valores más bajos se presentaron en los meses de junio, septiembre y octubre (Figura 3B). No se hallaron diferencias estadísticamente significativas basados en la abundancia promedio de caracoles, en los sectores estudio $(H=5.3, D F=2, p>0.05)$ y los meses de muestreo $(H=7.9, D F=7$, $\mathrm{p}>0.05$ ). En la época seca y lluviosa menor, se encontró un mayor número de individuos cerca al manglar en los estratos 1 y 2, mientras que, en la época lluviosa mayor los organismos se alejaron considerablemente de este ecosistema, principalmente en el estrato 3 (Figura 4).
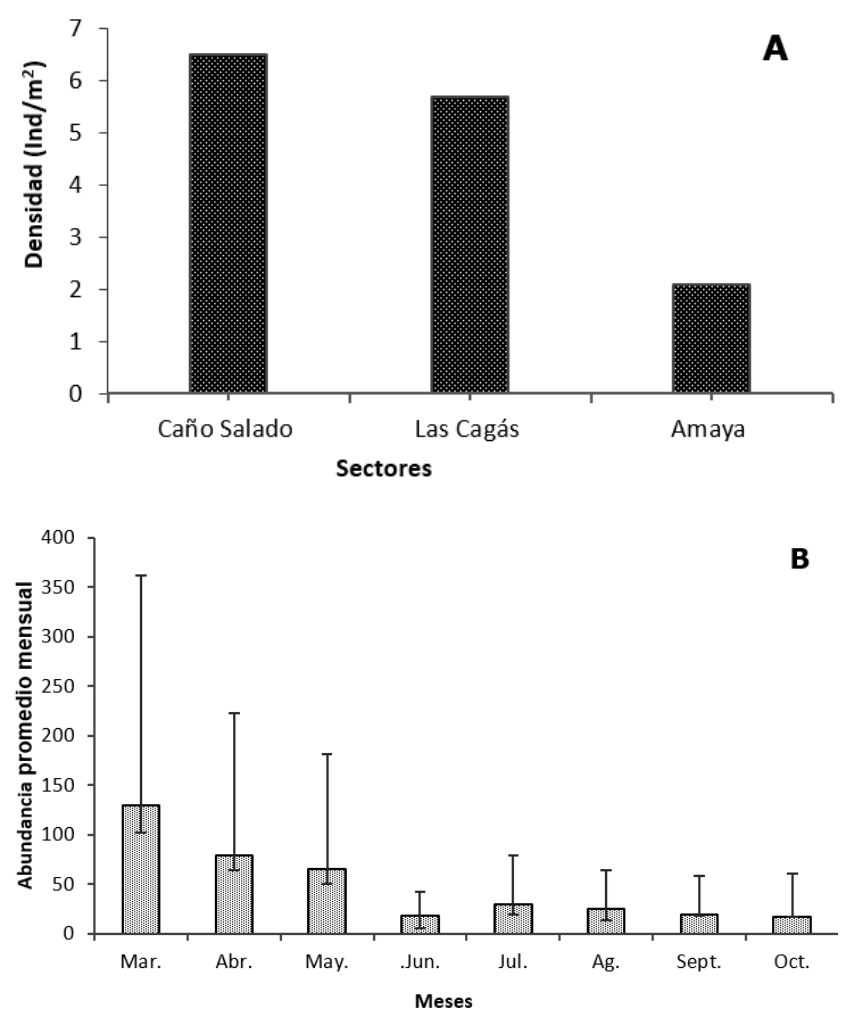

Figura 3. A. Densidad espacial de $M$. melongena en los sectores de estudio. B. Abundancia promedio mensual de $M$. melongena en la bahía de Cispatá, departamento de Córdoba, Caribe Colombiano.

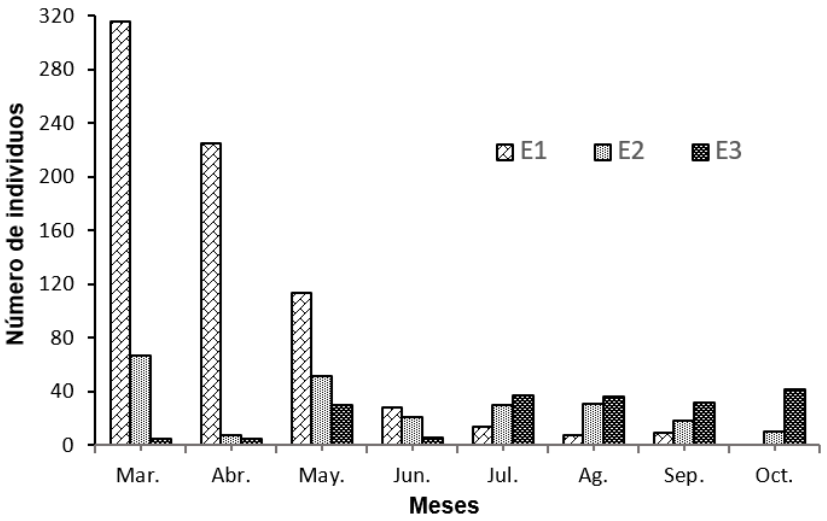

Figura 4.Número de individuos de M. melongena para los diferentes estratos de distancia con respecto al manglar.

En la bahía de Cispatá, el $63 \%$ de los individuos evaluados se encontraron en un intervalo de talla entre 41-61 mm, seguido del $20.6 \%$ $(20-40 \mathrm{~mm}), 15.8 \%(62-82 \mathrm{~mm})$ y el $0.5 \%$ (83-103 mm) respectivamente. La proporción sexual de $M$. melongena, fue de 1:1.2 (H:M), observándose un mayor número de machos que hembras durante el estudio. No obstante, la prueba de Chi-cuadrado indicó que no se encontraron diferencias estadísticamente significativas entre la proporción sexual y los sectores de captura $\left(X^{2}=5.7, p>0.05\right)$ y los meses de muestreo $\left(X^{2}=7.9, p>0.05\right)$. Las tallas mejor representadas en hembras fueron de 55 a $92 \mathrm{~mm}$ y en machos de 54 a $77 \mathrm{~mm}$ (Figura 5).

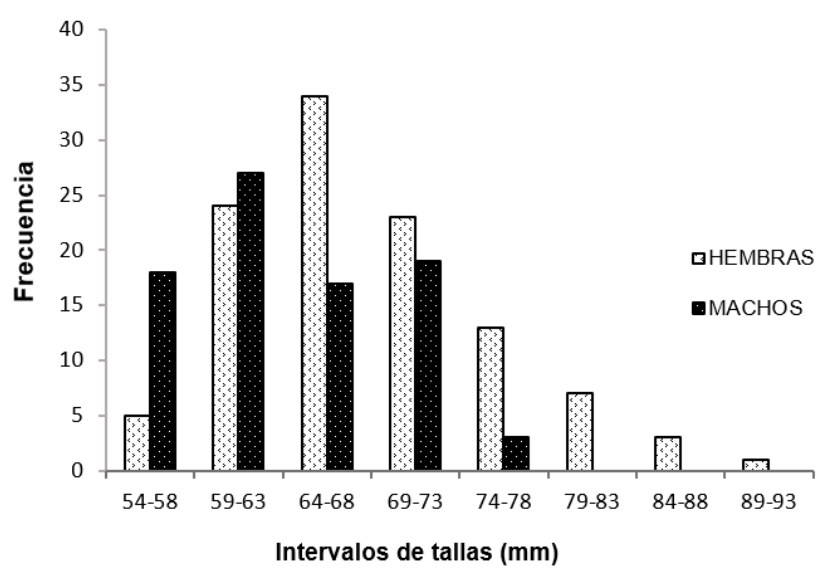

Figura 5.Distribución de frecuencias de tallas de machos y hembras de $M$. melongena en la bahía de Cispatá, departamento de Córdoba, Caribe Colombiano 
Efecto de las variables ambientales y la abundancia. El resultado del análisis de redundancia de las variables fisicoquímicas y de la abundancia de los individuos de $M$. melongena en los sectores y meses de muestreo se presenta en la figura 6. El primer eje del análisis del RDA explica un $68.1 \%$ de la varianza total, este eje esta correlacionado positivamente por la transparencia y negativamente por la salinidad y la temperatura. El segundo eje explica un $29.4 \%$ de la variabilidad total y esta correlacionado positivamente por los meses lluviosos (abril, junio, septiembre y octubre) y negativamente por marzo, julio y agosto. Los dos ejes del RDA explican el $\mathbf{9 7 . 5 \%}$ de la varianza acumulada.

Aunque las transparencia, temperatura y salinidad juegan un papel importante sobre las poblaciones de este caracol en área de estudio, las mayores abundancias de este caracol están positivamente correlacionadas en el mes de marzo con la salinidad, mientras que las menores abundancias están relacionadas con los meses lluviosos, así como con los descensos de la salinidad del agua (Figura 6).

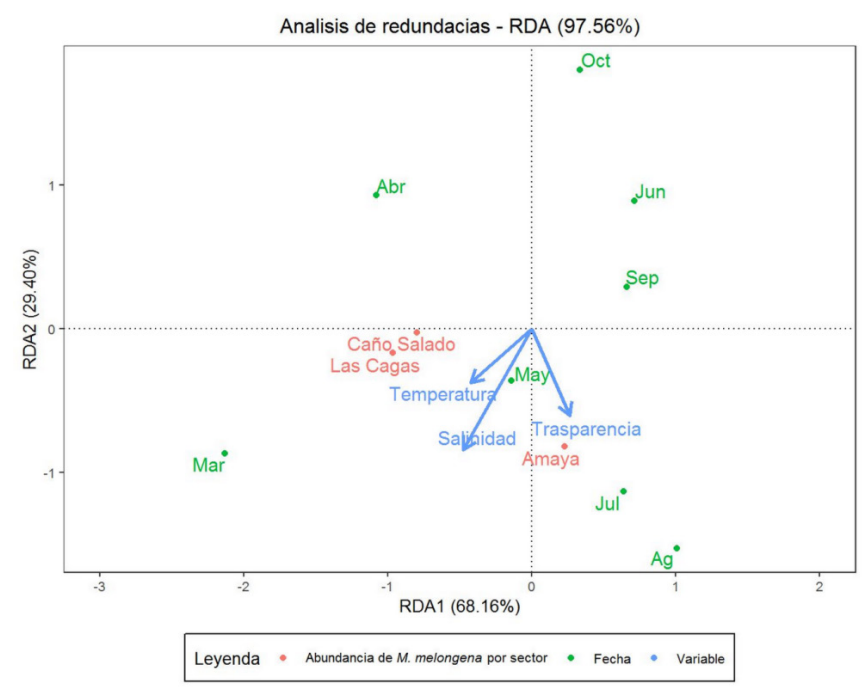

Figura 6. Análisis de redundancia (RDA) entre las variables fisicoquímicas y la abundancia de los individuos de M. melongena en los sectores y meses de muestreo

\section{DISCUSIÓN}

La temperatura del agua se mantuvo constante a lo largo de los meses de investigación, presentando una disminución en el mes de julio, lo que coincide con la época de transición. Este comportamiento es similar al reportado por Sánchez-Páez et al (8) y Ospina y Quintana-Saavedra (12) para el área de estudio, quienes indicaron que entre junio y julio esta variable tiende a descender a causa de los aumentos de la nubosidad. Del mismo modo, la transparencia alcanzo los máximos valores durante la época seca, mientras que los mínimos se obtuvieron durante la época Iluviosa mayor. Este resultado puede relacionarse con los niveles de precipitación, debido que, al incrementar las lluvias, aumenta la cantidad de material particulado, lo que a su vez influye en la turbidez del agua (12). La salinidad durante el mes de marzo presentó el máximo valor, debido al efecto de la intensa evaporación, mientras que los mínimos se obtuvieron en los meses de septiembre y octubre. Esta disminución de salinidad con el paso de los meses de estudio puede atribuirse al aumento en los aportes de aguas continentales con la llegada de las lluvias (12). El aumento de la salinidad coincidió con los altos valores en la abundancia de la especie. Esta relación es discutida por autores como Acosta et al (13), Kabir et al (14), Chávez et al (15) y Balán-Dzul y De Jesús-Navarrete (16), quienes corroboran que esta variable puede regular el ciclo reproductivo de ciertos gastrópodos.

La densidad de Melongena melongena se encontró por encima de la estimada por Hernández y Stotz (5) en el área de estudio, lo que sugiere una posible recuperación de la población atribuida a un descenso en el esfuerzo pequero por razones externas a la pesca como el desplazamiento de los pescadores a otras actividades como agrícolas, construcción de vías, obras civiles, entre otras. Por otro lado, el cambio en la dinámica hídrica del río Sinú ha favorecido la intrusión de aguas marinas en el complejo estuarino de la bahía, ocasionando un proceso de salinización de las aguas (8), situación que puede estar ayudando al aumento de las poblaciones en el área. Resultado que confirma lo registrado por Barroso y Matthews-Cascon (17), quienes reportaron que los cambios en los rangos de salinidad inciden en la abundancia de gastrópodos en un sistema estuarino.

Caño Salado fue el sector que registró la mayor abundancia de $M$. melongena, debido a la poca presión de este recurso por parte de los caracoleros, al ser considerada una zona de preservación en donde el aprovechamiento de los recursos hidrobiológicos es limitado (10); Las Cagas registró una abundancia importante, aunque por debajo de Caño Salado, esto como consecuencia del aprovechamiento racional del 
recurso que no llega a afectar la abundancia de la especie (8); Amaya mostró la menor abundancia, posiblemente relacionada con la poca disponibilidad de espacio y alimento para la especie, debido a que el sector no presenta un bosque de manglar consolidado, si no pequeños relictos de $R$. mangle. Según Cannicci et al (18) algunos caracoles estuarinos en el interior del bosque de manglar requieren condiciones específicas, siendo sensibles a la deforestación, así como a las alteraciones antropogénicas, cambiando su dinámica poblacional de manera inesperada, alterando el movimiento de las especies, la densidad poblacional, la dispersión de juveniles, la distribución espacial y el tamaño corporal.

La variación temporal de la población de $M$. melongena mostró un patrón influenciado por la precipitación. La abundancia fue mayor en marzo, durante la temporada seca y menor en septiembre y octubre, al inicio del segundo periodo lluvioso. Así la variabilidad en el número de individuos de la especie durante el año puede ser consecuencia de las diferencias de salinidad, así como la escasa demanda del recurso caracol, que obligo a los caracoleros a optar por otras fuentes de empleo. Autores como MolinaBolívar et al (19) y Quirós y Arias (20) señalan que en ecosistemas estuarinos inciden factores ambientales como las concentraciones de salinidad, temperatura y trasparencia, que son regulados por las temporadas de sequía y lluvias, condicionando la distribución y abundancia de las especies en los estuarios.

En cuanto a la distribución de $M$. melongena por estratos en la bahía de Cispatá, en los meses secos se observaron un mayor número de individuos próximos al manglar (estrato 1 y 2 ), mientras que en meses lluviosos estos se alejaron considerablemente del mismo (estrato 3 ), condición que pudo atribuirse a las variaciones de salinidad durante estos periodos, ya que en época seca reducen los flujos hídricos fluviales, ocasionando elevados valores de salinidad, debido a la intensa evaporación. Mientras que, en época lluviosa, se presentan menores valores en la concentración de sales disueltas, esto influenciado por el mayor aporte fluvial producido por las altas precipitaciones y aumento en el caudal del río Sinú (12). De acuerdo con Ortiz y Blanco (21), esta conducta es característica de las especies estuarinas las cuales tienden a fluctuar ampliamente en su abundancia debido a las variaciones de las condiciones en las masas de agua. Adicionalmente, Acosta et al
(13) y Mieszkowska at al (22) señalan que los incrementos en la salinidad provocan cambios en la estructura poblacional de algunos gastrópodos. Además, estos resultados confirman lo registrado por Kabir et al (14), quien reportó que esta variable es uno de los parámetros ambientales que tiene mayor influencia en la distribución de caracoles estuarinos con respecto a las zonas de manglar.

La gran mayoría de individuos que componen las poblaciones de $M$. melongena en la bahía de Cispata son de talla mediana (longitud de la concha entre 41-61 mm), lo cual parece indicar elevadas tasas de reclutamiento o la existencia de algún factor de mortalidad que afecta preferencialmente a los animales más grandes (18). También, la presión por extracción que se ejerce mayormente sobre los individuos de tallas más grandes es aparentemente uno de los principales responsables de la dominancia de individuos medianos en las poblaciones de melongénidos en el área de estudio. Por otro lado, M. melongena no presentó diferencias estadísticamente significativas en la proporción sexual $(H: M)$ y las hembras fueron mayores que los machos. Estudios poblacionales de moluscos con otros melongénidos realizados por Santos et al (11) y Young et al (23), describieron un patrón similar al del presente estudio, con presencia de hembras entre los individuos de mayor talla y machos entre los de menor talla. Así, la igualdad de la proporción entre hembras y machos con la talla podría explicarse por un crecimiento diferencial entre sexos, donde las hembras alcanzan tallas mayores con el fin de acumular alimento para la reproducción, mientras que los machos invierten sus reservas en reproducirse, por lo que no gastan energía en aumentar su tamaño $(11,14,24)$.

En conclusión, la población de $M$. melongena en la bahía de Cispatá ha presentado signos de recuperación en los últimos años tomando como referencia el trabajo de Hernández y Stotz (5), debido a que la mayoría de los individuos registrados han alcanzado un intervalo de talla entre 41-61 $\mathrm{mm}$, el cual parece indicar que la especie está alcanzando la TMM. La proporción sexual (H:M) no mostró diferencias estadísticamente significativas, lo que indica claramente que en Cispatá en el departamento de Córdoba, la tendencia es hacia una proporción igual $1: 1(\mathrm{H}: \mathrm{M})$. La distribución de $M$. melongena en el área de estudio está influenciada por los periodos hidrológicos, donde la salinidad fue la variable que más influyó en la abundancia de 
Niño-Miranda et al - Estado actual de M. melongena en Cispatá

la especie. Por lo cual, se recomienda seguir realizando estudios que incluyan otras variables abióticas y bióticas en los sectores en donde se realiza extracción de este recurso, con el fin de seguir evidenciando posibles cambios positivos y/o negativos en el comportamiento de sus poblaciones. Del mismo modo, es importante realizar investigaciones donde se evalúen los aspectos pesqueros de la especie, debido al interés comercial que esta especie representa para la región, y finalmente desarrollar campañas de concientización con los caracoleros de la zona, con el fin de concertar medidas de manejo y uso para un aprovechamiento sustentable.

\section{Conflicto de intereses}

Los autores de presente estudio declaramos que no existe conflicto de intereses con la publicación del manuscrito.

\section{Agradecimientos}

Esta investigación hace parte del macroproyecto sobre diversidad de invertebrados en Córdoba, financiado por la Universidad de Córdoba. Agradecemos a la Asociación de Caimaneros (ASOCAIMAN) por toda su colaboración y apoyo en la realización de la presente investigación. A la Dra. Clara Sierra Díaz por el apoyo logístico brindado. A Manuel Sánchez Crespo, Sandra Hernández Barrero y Nancy Suarez Mozo por sus valiosos aportes durante la investigación. Finalmente, agradecemos al docente Robinson Rosado Cárcamo por su confianza en todo este proceso.

\section{REFERENCIAS}

1. Castillo-Rodríguez Z. Biodiversidad de moluscos marinos en México. Rev Mex Biodivers. 2014; 85(supl.1):419-430. https://doi.org/10.7550/rmb.33003

2. Quirós J, Campos NH. Moluscos asociados a ensamblajes macroalgales en el litoral rocoso de Córdoba, Caribe Colombiano. Bol Invest Mar Cost. 2013; 42(1):101120. http://dx.doi.org/10.25268/bimc. invemar.2013.42.1.62

3. Álvarez-León R. Biodiversidad de la flora y fauna asociada a los manglares de Colombia. Arq Ciên Mar. 2015; 48(2):85-92. https:// doi.org/10.32360/acmar.v48i2.5852

4. López-Sánchez CM, Mancera-Pineda JE. Parámetros estructurales de dos poblaciones de Crassostrea rhizophorae (Ostreidae) en bahía de Cispatá, Caribe Colombiano. Acta Biol Colomb. 2019; 24(2):361-371. http:// dx.doi.org/10.15446/abc.v24n2.68941

5. Hernández S, Stotz WB. Reproductive Biology of the "Copey" snail Melongena melongena (Linnaeus, 1758) in Cispatá Bay on the Caribbean coast of Colombia. J Shellfish Res. 2004; 23(3):849-854. https://go.gale.com/ps/anonymous?id=G ALE\%7CA130777671\&sid=googleScholar\& $\mathrm{v}=2.1$ \&it $=r \&$ linkaccess $=$ abs\&issn $=073080$ $\underline{00 \& p=A O N E \& S W=W}$
6. Díaz J, Puyana M. Moluscos del Caribe colombiano: un catálogo ilustrado. Bogotá, Colombia: COLCIENCIAS, Fundación Natura e INVEMAR; 1994.

7. Nieto-Ramón B, Chasqui L, Rodríguez AM, Castro E, Gil-Agudelo D. Composición, abundancia y distribución de las poblaciones de gasterópodos de importancia comercial en La Guajira, Caribe colombiano. Rev Biol Trop. 2013; 61(2):683-700. https://doi. org/10.15517/rbt.v61i2.11168

8. Sánchez-Páez H, Ulloa H, Tavera H, Gil W. Plan de manejo integral de los manglares de la zona de usos sostenible del sector estuarino de la bahía de Cispatá, departamento de Córdoba -Colombia. Bogotá: Corporación Autónoma Regional de los Valles del Sinú y del San Jorge (CVS), Corporación Nacional de Investigación y Fomento Forestal (CONIF); 2005. https:// es.scribd.com/document/110384331/Plande-Manejo-Integral-de-los-Manglares-dela-Zona-de-Uso-Sostenible-de-la-Bahia-deCispata-Colombia

9. Duarte L, Díaz-Vesga R, Cuello F, Manjarrés L. Cambio estacional en la fauna acompañante de la pesquería estacional de arrastre de camarón del golfo de Salamanca, Mar Caribe de Colombia. Acta biol Colomb. 2013; 18(2):319-328. https://revistas. unal.edu.co/index.php/actabiol/article/ view/37604/40734 
10. Corporación Autónoma Regional de Los Valles del Sinú y del San Jorge - CVS, Instituto de Investigaciones Marinas y Costeras - INVEMAR. Plan Integral de manejo del Distrito de Manejo Integrado (DMI) bahía de Cispatá - La Balsa - Tinajones y sectores aledaños del delta estuarino del río Sinú, departamento de Córdoba. Serie de Publicaciones Especiales No. 18. Santa Marta, Colombia: INVEMAR; 2010. https://www.oceandocs.org/bitstream/ handle/1834/6607/Plan manejo DMI Cispata.pdf?sequence $=1$ \&isAllowed $=y$

11. Santos J, Enríquez $M$, Aldana D. Dinámica poblacional y reproductiva de Turbinella angulata y Busycon perversum (Mesogasteropoda: Turbinellidae y Melongenidae) en el banco de Campeche, México. Rev Biol Trop. 2013; 61(1):15-28. https://doi.org/10.15517/rbt.v61i1.10940

12. Ospina L, Quintana-Saavedra DM. Variación espacio-temporal de la calidad del agua del golfo de Morrosquillo durante al año 2013. Bol Cient CIOH 2015; 33(1):19-38. https:// www.cioh.org.co/dev/publicaciones/ acceso dev.php?nbol=cioh bcc3301.pdf

13. Acosta V, Betancourt R, Prieto A. Estructura comunitaria de bivalvos y gasterópodos en raíces de mangle rojo Rhizophora mangle (Rhizophoraceae) en isla Larga, bahía de Mochima, Venezuela. Rev Biol Trop. 2014; 62(2):551-565. https://doi.org/10.15517/ rbt.v62i2.9803

14. Kabir M, Abolfathi M, Hajimoradloo A, Zahedi S, Kathiresan K, Goli S. Effect of mangroves on distribution, diversity and abundance of molluscs in mangrove ecosystem: a review. AACL Bioflux 2014; 7(4):286-300. http:// www.bioflux.com.ro/docs/2014.286-300.pdf

15. Chávez J, Enríquez M, Aldana D. Abundancia y diversidad larval de gasterópodos en el Caribe Mexicano en relación con la temperatura, la salinidad y el oxígeno disuelto. Rev Biol Trop. 2014; 62(Suppl. 3):223-230. https://doi.org/10.15517/RBT. V6210.15917

16. Balán-Dzul V, De Jesús-Navarrete $A$. Densidad, abundancia y estructura poblacional del caracol blanco Strombus costatus en el Caribe Mexicano. Rev Biol Mar Oceanogr. 2011; 46(1):1-8. http://dx.doi. org/10.4067/S0718-19572011000100001
17. Barroso CX, Matthews-Cascon H. Distribuição espacial e temporal da malacofauna no estuário do rio Ceara, Ceara, Brasil. Pan-Am J Aquat Sci. 2009; 4(1):7986. https://panamjas.org/pdf artigos/ PANAMJAS 4(1) 79-86.pdf

18. Cannicci S, Burrows D, Fratini S, Smith III TJ, Offenberg J, Dahdouh-Guebas F. Faunal impact on vegetation structure and ecosystem function in mangrove forests: A review. Aquat Bot. 2008; 89(2):186-200. https://doi.org/10.1016/j. aquabot.2008.01.009

19. Molina-Bolívar G, Jiménez IA, Nava ML. Taxocenosis Mollusca-Crustacea en raíces de Rhizophora mangle, delta del río Ranchería - La Guajira, Colombia. Intropica. 2017; 12(2):87-100. https:// doi.org/10.21676/23897864.2281

20. Quirós J, Arias J. Taxocenosis de moluscos y crustáceos en raíces de Rhizophora mangle (Rhizophoraceae) en la bahía de Cispatá, Córdoba, Colombia. Acta Biol Colomb. 2013; 18(2):329-340. https://revistas. unal.edu.co/index.php/actabiol/article/ view/37794/40857

21. Ortiz LF, Blanco JF. Distribución de los gasterópodos del manglar, Neritina virgínea (Neritidae) y Littoraria angulifera (Littorinidae) en la Ecorregión Darién, Caribe colombiano. Rev Biol Trop. 2012; 60(1):219-232. https://doi.org/10.15517/ RBT.V60I1.2755

22. Mieszkowska N, Hawkins SJ, Burrows MT, Kendall MA. Long-term changes in the geographic distribution and population structure of Osilinus lineatus (Gastropoda: Trochidae) in Britain and Ireland. J Mar Biol Assoc. U.K. 2007; 87:537-545. https://doi. org/10.1017/S0025315407053799

23. Young JM, Yeiser BG, Whittington JA. Spatiotemporal dynamics of spawning aggregations of common snook on the east coast of Florida. Mar Ecol Prog Ser. 2014; 505:227-240. https://doi.org/10.3354/ meps 10774

24. Kandeel KE, Mohammed SZ, Mostafa AM, Abd-Alla ME. Reproductive biology of the cockle Cerastoderma glaucum (Bivalvia: Cardiidae) from Lake Qarun, Egypt. Egypt J Aquat Res. 2013; 39(4):249-260. https:// doi.org/10.1016/j.ejar.2013.12.003 